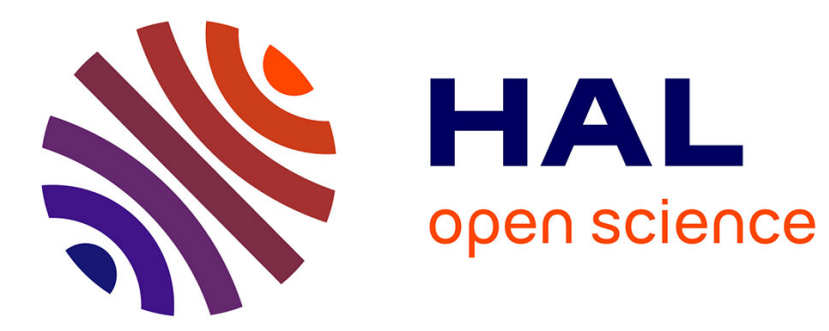

\title{
The frozen state in the liquid phase of side-chain liquid-crystal polymers
}

\author{
H. Mendil, Laurence Noirez, P. Baroni, I. Grillo
}

\section{To cite this version:}

H. Mendil, Laurence Noirez, P. Baroni, I. Grillo. The frozen state in the liquid phase of sidechain liquid-crystal polymers. Physical Review Letters, 2006, 96, pp.077801. 10.1103/PhysRevLett.96.077801 . hal-01361941

\section{HAL Id: hal-01361941 \\ https://hal.science/hal-01361941}

Submitted on 7 Sep 2016

HAL is a multi-disciplinary open access archive for the deposit and dissemination of scientific research documents, whether they are published or not. The documents may come from teaching and research institutions in France or abroad, or from public or private research centers.
L'archive ouverte pluridisciplinaire HAL, est destinée au dépôt et à la diffusion de documents scientifiques de niveau recherche, publiés ou non, émanant des établissements d'enseignement et de recherche français ou étrangers, des laboratoires publics ou privés. 


\title{
The Frozen State in the Liquid Phase of Side-Chain Liquid-Crystal Polymers
}

\author{
H. Mendil, L. Noirez, ${ }^{*}$ and P. Baroni \\ Laboratoire Léon Brillouin (CEA-CNRS), Ce-Saclay, 91191 Gif-sur-Yvette Cédex, France \\ I. Grillo \\ Institut Laue Langevin, BP 156, 38042 Grenoble Cédex 09, France
}

(Received 27 May 2005; revised manuscript received 2 December 2005; published 24 February 2006)

\begin{abstract}
Quenched isotropic melts of side-chain liquid-crystal polymers reveal surprisingly an anisotropic polymer conformation. This small-angle neutron-scattering (SANS) result is consistent with the identification of a macroscopic, solidlike response in the isotropic phase. Both experiments (rheology and SANS) indicate that the polymer system appears frozen on millimeter length scales and at the time scales of the observation. This result implies that the flow behavior is not the terminal behavior and that crosslinks or entanglements are not a necessary condition to provide elasticity in melts.
\end{abstract}

Liquids are known as weakly correlated systems. On cooling, glass-forming liquids transit to a strongly correlated glassy state. Nevertheless, the frontier between the liquid and solid states remains obscure; our experiments show that so far unidentified gigantic solid correlations exist far away from glass and phase transitions, in the "molten" state of side-chain liquid-crystal polymers (LC polymers). We thus show that polymers are solidlike far away from any phase or glass transition. This surprising finding is, however, consistent with the mode coupling developments (MCT) [1], the Fischer's clusters approach [2], or extended NMR experiments [3]. Such insights also provide a better understanding of unexpected flow behaviors in complex fluids [4] as the spectacular shear-induced phase transition in LC polymers [5]. Indeed, these shearinduced effects observed far away from any phase transition can be explained using neither the conventional viscoelastic concept (Rouse, reptation time) nor a conventional pretransitional coupling [6]. Time scales longer [7] than the individual polymer dynamics are involved and must be identified.

We present here two original experiments that allow one to identify these slow relaxation modes and lead us to the fundamental result: the LC polymer is solidlike far from any phase or glass transition.

Because of the coupling between the main chain and the mesogenic side group, LC polymers display various anisotropic main-chain conformations in the liquid-crystal phases [8]. Oblate or prolate chain anisotropies have been found depending on the symmetry of the phase and on the order parameter [9]. In the isotropic phase, the longrange liquid crystalline order is lost, giving rise to a macroscopically averaged isotropic main-chain conformation. We demonstrate here that it is possible to identify a chain anisotropy within the isotropic phase. This is our first result, obtained by decoupling the fast phase dynamics from the slow melt time scales. It reveals the persistence of long nonrelaxed melt time scales. The second funda- mental result is the unambiguous observation of a solidlike dynamic behavior within the isotropic phase. This contrasts with the expected flow behavior [10] and reveals that without cross-links or entanglements, LC polymers display elasticity. Both observations indicate millimeter-scale solidlike behavior. This so far unidentified macroscopic huge melt cohesion in a supposed "liquid" state has multiple consequences both from a theoretical and an experimental point of view. For example, the previously reported shearinduced phenomena [5] must be considered as the result of the solicitation of a gel and the conventional description (pretransitional orientational-order fluctuations, viscoelastic relaxation time) should be revisited. Finally, our observations together with the "Fischer's clusters" light scattering approach [2], Martinoty's piezorheometer measurements [11], and the MCT that predicts a crossover from liquid to glass behavior at about 50 to $80 \mathrm{~K}$ above the calorimetric glass transition temperature $T_{g}[1,2]$ provide firm indications that long-range solid correlations persist even far away from $T_{g}$.

The strategy adopted is the following. In the first part of the Letter, we examine the effect of nonequilibrium thermal treatments on the polymer conformation. We identify a persistence of the chain anisotropy in the quenched isotropic phase, which reveals nonrelaxed long time scales. In the second part of the Letter, we examine the viscoelastic behavior. We identify a solidlike behavior and analyze this elastic response versus temperature.

The LC polymer used to illustrate our purpose is a methoxy-phenyl benzoate substituted polyacrylate [12] synthesized at the Laboratoire:

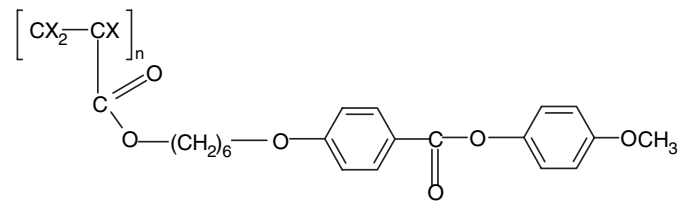

where $X=\mathrm{H}$ or D. 
The weight average molecular weight of the 1:1 hydrogenated and deuterated polymer mixture is $30200 \mathrm{~g} \cdot \mathrm{mol}^{-1}$ and the polydispersity index is 2.3 (determined by size exclusion chromatography-light scattering on line). The corresponding polymerization degree of 85 indicates an unentangled melt (polybutylacrylate chains are entangled from polymerization degrees above 340 , and higher when the side-chain sterical hindrance is reinforced). The polymer displays the following mesophases: $T_{g}-22^{\circ} \mathrm{C}-\mathrm{S}_{\mathrm{A} 1}$ (smectic) $-89^{\circ} \mathrm{C}-\mathrm{N}$ (nematic) $-116^{\circ} \mathrm{C}$ I(isotropic).

The dimensions of the polymer main chain are determined using the small-angle neutron-scattering (SANS) technique. The polymer is placed between two $1 \mathrm{~mm}$ thick quartz disks of $12 \mathrm{~mm}$ diameter separated by $1 \mathrm{~mm}$ sample thickness. The same sample is studied in all the experiments [rheology, SANS at the LLB (PAXY) and at the ILL (D22)].

The SANS technique requires the preparation of a mixture of hydrogenated polymers with polymers deuterated on the main chain. The signal measured under the Guinier approximation conditions, $S(q)$, is proportional to the form factor of the main-chain part of the polymer [13]. In the $(x O y)$ plane, $O x$ and $O y$ being the axes parallel and perpendicular to the director, respectively: $S(q) \alpha 1 /\langle 1+$ $\left.\left(q_{x}^{2} R_{x}^{2}+q_{y}^{2} R_{y}^{2}\right)\right\rangle$ where $q$ the scattering vector $[q=$ $4 \pi / \lambda \sin (\theta / 2)$ with $q$ the scattering angle]. The components, $R_{x}=R_{z}, R_{y}$, of the radius of gyration of the polymer main chain following the two axes are deduced from a $2 D$ treatment of the signal [14].

Under thermodynamic equilibrium conditions, the evolution of the polymer conformation (detailed in [12]) is displayed in Fig. 1 (the macroscopic alignment of the mesophases is ensured by applying a $1.2 \mathrm{~T}$ magnetic field). The main-chain radius of gyration along and perpendicular to the director are noted $\left(R_{\|}\right)$and $\left(R_{\perp}\right)$, respectively. In the equilibrium isotropic phase, no anisotropy is observed. Below the isotropic-nematic transition, $105<\mathrm{T}<$

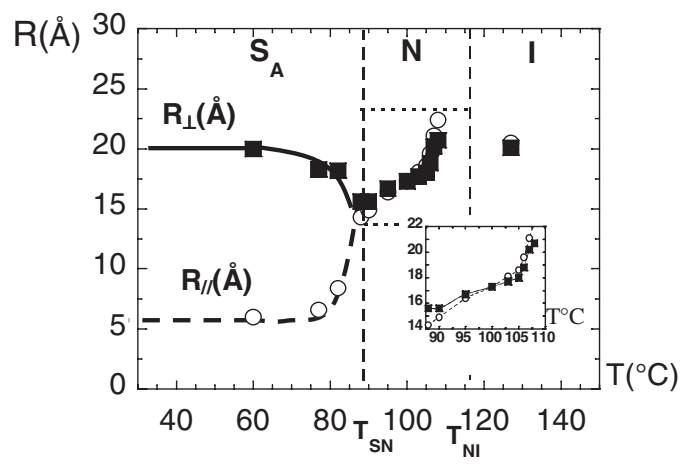

FIG. 1. Radius of gyration of the polymer main chain parallel and perpendicular to the director $\left(R_{\|}\right.$and $R_{\perp}$, respectively) versus temperature (PAXY data: sample-detector distance, $1.5 \mathrm{~m}$; wavelength, $10 \AA$ ). The dotted lines indicate the smectic to nematic and the nematic to isotropic transition temperatures. The inset displays $R_{\|}$and $R_{\perp}$ in the nematic phase. $116^{\circ} \mathrm{C}$, one can detect a very weak prolate tendency $\left(R_{\|} / R_{\perp}=2 \%\right)$. Above the smectic-nematic transition, the shape is weakly oblate $\left(R_{\|} \leq R_{\perp}\right)$. Finally, the oblate anisotropy in the smectic phase $\left(R_{\perp} / R_{\perp} \approx 400 \%\right)$ corresponds to a main-chain confinement between mesogen layers [Fig. 2(a)] [15].

In the nonequilibrium conditions, the sample is heated, without magnetic field, from the glassy state by direct contact with a hot stage, at $20^{\circ} \mathrm{C}$ above the nematicisotropic transition temperature (scheme of Fig. 2). The cell is not sealed and thus not geometrically constrained. The contact time is fixed at $45 \mathrm{~s}$ to ensure a total transit into the isotropic state (this corresponds to a temperature gradient of at least $145^{\circ} \mathrm{C} \cdot \mathrm{min}^{-1}$ from room temperature). The sample is then directly thrown in a liquid nitrogen dewar. The heating time is very long compared to the molecular liquid-crystal dynamics. After this thermal process, the liquid-crystal ordering is suppressed. It can be checked that the sample (in its glassy state at room temperature) has been quenched in the isotropic phase because of its optical transparency, the absence of birefringence between crossed polarizers, and the absence of structure at large scattering angles. Figure 2(a) displays the SANS signal (I) at room temperature before the thermal treatment (reference); the main chains are extended perpendicular to the director $\left(R_{\|} \ll R_{\perp}\right)$ as described above (Fig. 1). After this thermal treatment, the SANS signal corresponding to
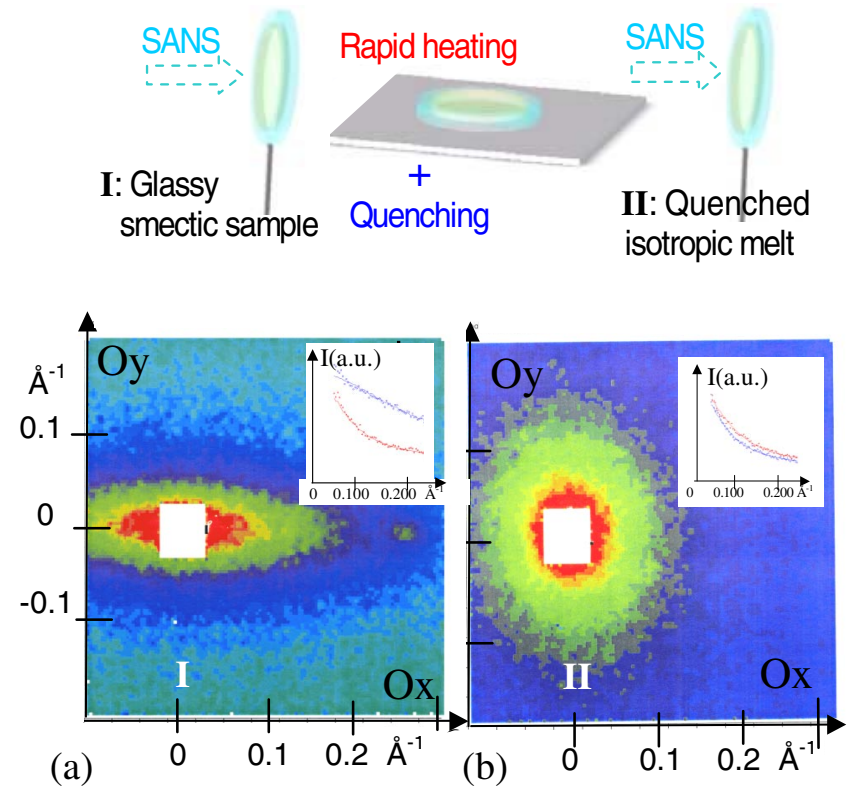

FIG. 2 (color online). Scheme of the thermal protocol and 2D neutron-scattering patterns (D22 data: sample-detector distance, $2.55 \mathrm{~m}$; wavelength, $6 \AA$ ). The director is parallel to $O x$. The color palette corresponds to intensity levels. The insets display the scattering curves and the corresponding fits along (red curve) and perpendicular to (blue curve) the director. (a) Reference at room temperature (I). The 001 smectic reflection can be noticed at $q=2 \pi / 25 \AA$. (b) After the nonequilibrium thermal cycle (quenched from the isotropic phase) (II). 
the quenched isotropic phase (II) does not display an isotropic chain conformation. In contrast, a spectacular inversion from its initial smectic oblate conformation to a singular prolate conformation is observed: $R_{\|} \gg R_{\perp}$. This anisotropy does not correspond to any equilibrium conformations; the rate $R_{\|} / R_{\perp}=12 \%$ is significantly larger (and underestimated) than any prolate conformation observed at equilibrium (the highest prolate tendency was estimated at $2 \%$ at $105-115^{\circ} \mathrm{C}-$ Fig. 1). Finally, at the second thermal treatment, the isotropic conformation is restored.

We explain the singular anisotropy as resulting from the kinetic superimposition of the thermal and stress history from the smectic up to the isotropic phase. During this process, the chains undergo a succession of conformational changes imposed by the evolution of the liquid-crystal field. During the transition from the smectic to the nematic arrangement, the positional order is transformed into an orientational order, this symmetry change implies a translation motion of the mesogens with respect to the others. Such a molecular shearing favors the prolate polymer shape. The experiment shows that $45 \mathrm{~s}$ is not sufficient for the chains to relax from the rapid thermal crossing of the mesophases and to release the corresponding intermolecular stresses. Long relaxation time scales $(>35 \mathrm{~s}$ at least) are necessarily involved in the LC polymer dynamics. What provides this spectacular memory effect?

We perform rheological measurements to characterize, in particular, in the isotropic phase, the characteristic times of these polymer melts. These experiments are carried out using an ARES rheometer equipped with an air-pulsed oven used in dynamic frequency sweep mode. The sample is left in a vacuum chamber for several hours while heated far above the glass transition to remove entrapped gas bubbles. The sample is then placed between plate-plate (diameter $12 \mathrm{~mm}$ ) fixtures.

Since our study is mainly focused on the dynamics in the isotropic phase, ordinary polymer melts will be our reference. The conventional terminal behavior of ordinary polymers in the molten state (at $T>T_{g}$ ) is the flow behavior, commonly described by the Maxwell model. This describes the fact that above a characteristic viscoelastic time, the "terminal time," the polymer is supposed to flow as a liquid. The upper inset of Fig. 3 illustrates the conventional low frequency response (flow behavior). In this regime, $G^{\prime}$ and $G^{\prime \prime}$ fit with $\omega^{2}$ and $\omega$ scaling, respectively $\left(G^{\prime} \ll G^{\prime \prime}\right)$, with the intersection of $G^{\prime}$ and $G^{\prime \prime}$ defining the characteristic terminal time. In contrast, we show by avoiding the polymer-substrate slipping (strong anchoring and small strain amplitudes are necessary conditions) that the nondissipative rheological LC-polymer response is actually solidlike and not the flow behavior $\left(G^{\prime} \gg G^{\prime \prime}\right.$ with $G^{\prime}$ and $G^{\prime \prime}$ constant).

Figure 3 illustrates the frequency dependence $G^{\prime}(\omega)$ and $G^{\prime \prime}(\omega)$ of the polymer at $\Delta T=+14^{\circ} \mathrm{C}$ over the isotropicnematic transition in the linear regime and in the full

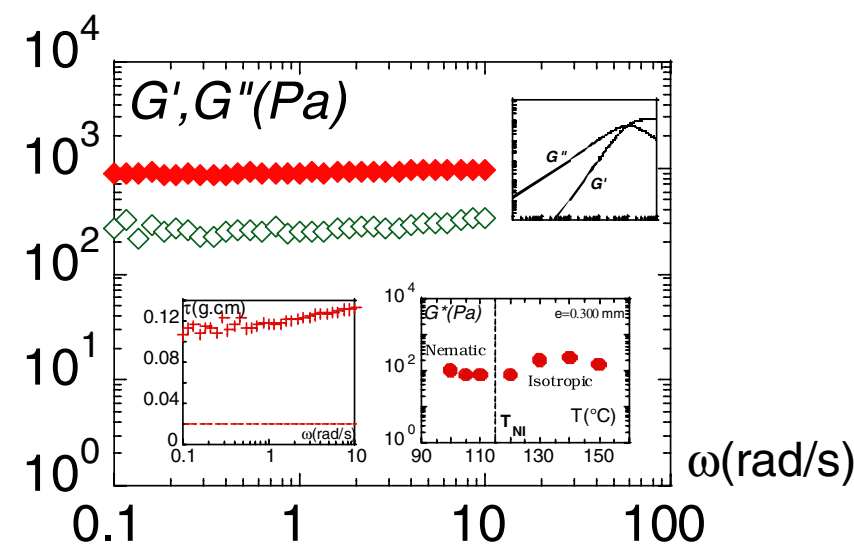

FIG. 3 (color online). Evolution of the elastic $G^{\prime}(\diamond)$ and loss $G^{\prime \prime}(\diamond)$ moduli versus frequency at $2.5 \%$ deformation (linear regime), of the LC polymer in the isotropic phase $\left(T=130^{\circ} \mathrm{C}\right.$, $\Delta T=+14{ }^{\circ} \mathrm{C}$, plate-plate geometry, $0.150 \mathrm{~mm}$ gap thickness). The left inset displays the torque $\tau(\mathrm{g} \cdot \mathrm{cm})$ together with the sensitivity limit of the device (dashed line). The lower right inset displays the evolution of the dynamic complex modulus $G^{*}$ as a function of the temperature in the linear regime $(0.300 \mathrm{~mm}$ gap thickness). The upper right inset displays for comparison the terminal flow behavior [as predicted for uncross-linked polymers (Maxwell model)].

sensitivity range of the torque (left inset of Fig. 3). An elastic modulus independent of the frequency defining an elastic plateau is revealed. The loss modulus $G^{\prime \prime}(\omega)$ is negligible compared to the elastic modulus $G^{\prime}$. The elastic component merges with the complex modulus $G^{*}$ value; the system displays solidlike behavior in a wide frequency range $\left(10^{-1}-10 \mathrm{rd} / \mathrm{s}\right)$, with a modulus comparable to the rubbery plateau of entangled polymers [16].

These results are very surprising for both reasons: $G^{\prime}$ is higher than $G^{\prime \prime}$, and a solidlike behavior is measured in a frequency region where polymer melts are expected to flow (at least at $T-T_{g}=+100^{\circ} \mathrm{C}, T-T_{\mathrm{NI}}=+14{ }^{\circ} \mathrm{C}$ ).

The lower right inset of Fig. 3 displays the evolution of the complex modulus $G^{*}=\left(G^{\prime 2}+G^{\prime 2}\right)^{1 / 2}$ versus temperature in the linear regime. The temperature window ranges over $60^{\circ} \mathrm{C}$ and crosses the isotropic-nematic transition temperature. Temperatures over $160^{\circ} \mathrm{C}$ were not tempted because of a possible thermal degradation of the acrylate ester [17]. We also do not report on the behavior below the smectic-nematic transition, since, when the smectic order is established, the elastic modulus becomes strongly coupled with the elasticity of the positional smectic order as already reported [18]. This inset shows that the elastic plateau is not clearly sensitive to the temperature, and is independent of the isotropic-nematic transition $\left(T_{\mathrm{NI}}=116 \pm 0.5^{\circ} \mathrm{C}\right)$. The elasticity does not originate from mesomorphic properties and is not reminiscent from phase pretransitional dynamics. It is a melt property, which can be measured even $100{ }^{\circ} \mathrm{C}$ above the glass transition.

These experimental observations lead to the conclusion that a classical approach (Rouse, reptation models) does 
not describe the behavior of LC-polymer melts. Clearly, a connectivity between chains provides the necessary cohesion, whereas the molecular weight and the dimensions of the chain $\left(R_{g} \approx 34 \AA\right)$ indicate that the polymer is unentangled. We believe that the polymer architecture plays a key role; the side-branch mesogens should act as effective interconnection between the chains. The interconnection effect is reinforced by the degree of interdigitation (steric effect, van der Waals interactions), the side-branch interaction (polar effect, in particular, in liquid-crystal polymers), the grafting density, and also the degree of polymerization. These different parameters should interplay and determine the resulting elasticity.

This conclusion is also consistent with the primary observations of long relaxation time scales [2] far from the glass transition in glass-forming systems, theoretically explained in terms either of the coexistence of transient orientational and positional order [1,19] or of frozen density fluctuations [20]. From piezorheometer measurements on thin films [11], it was proposed that the polymer melt exhibits dynamic micron-sized heterogeneities ("clusters"). We show here by using a conventional rheometer (sample thickness $0.300 \mathrm{~mm}$ ) and from the SANS results (1 mm thickness) that the elasticity persists independently of the temperature at much larger scales; in contrast to the clusters assumption [11], LC polymers are macroscopically solidlike materials.

Finally, it is useful to notice that we do not use a particular polymer or particular liquid crystal; other LC polymers give rise to similar results. The study is now extended to other chemical compounds [7]. We propose that the individual polymer chain motion is synchronized to each other via the side-chain (mesogen) interconnection. This results in a three-dimensional connectivity; a macroscopic physical gel in which solid-state dynamic effects are measurable by the elastic plateau and indirectly by the nonequilibrium effects described above. The concept of mesogens acting as effective cross-linkers is also consistent with the singular flow behavior of the smectic phase of LC polymers. Indeed, surprisingly the smectic layer thickness decreases under flow [21]. This is interpreted by a layer tilting. Such a transition requires interconnecting layers. This has never been observed for the low molecular weight liquid crystals; in contrast, layer instabilities are expected to increase the layer thickness [22]. To conclude, we have shown unambiguously that it is possible to reveal millimeter-scale elastic properties in LC polymer, without the contribution of any entanglement (both the backbone and the side groups are too short or too rigid to allow any entanglement). This experimental observation leads to the conclusion that the individual chains are connected dynamically, creating a giant macroscopic elastic interconnection, which persists far above the glass and phase transitions. We trust that the identification of this novel type of elasticity will trigger various experimental and theoretical work to understand the true nature of polymers and to predict their properties.

*Electronic address: noirez@llb.saclay.cea.fr

[1] W. Götze, in Liquids, Freezing and Glass Transition, Proceedings of the Les Houches Workshop, edited by J.-P. Hansen et al. (North-Holland, Amsterdam, 1989), pp. 287-504; W. Götze and L. Sjögren, Rep. Prog. Phys. 55, 241 (1992); H. R. Brand and K. Kawazaki, J. Phys. II (France) 4, 543 (1994); Physica (Amsterdam) 324A, 484 (2003); D. R. Reichman and P. Charbonneau, J. Stat. Mech. (2005) P05013.

[2] E. W. Fischer, Physica (Amsterdam) 201A, 183 (1993); E. W. Fischer et al., Acta Polym. 45, 137 (1994); E. W. Fischer et al., J. Non-Cryst. Solids 307, 584 (2002).

[3] K. Schmidt-Rohr and H. W. Spiess, Phys. Rev. Lett. 66, 3020 (1991).

[4] J. F. Berret, D. C. Roux, G. Porte, and Linder, Europhys. Lett. 25, 521 (1994); V. Schmitt, F. Lequeux, A. Pousse, and D. Roux, Langmuir 10, 955 (1994).

[5] C. Pujolle-Robic and L. Noirez, Nature (London) 167, 409 (2001).

[6] S. Hess, Z. Naturforsch. 31a, 1507 (1976); A. Onuki and K. Kawasaki, Ann. Phys. (N.Y.) 121, 456 (1979); P. D. Olmsted and P. Goldbard, Phys. Rev. A 41, 4578 (1990); H. Pleiner, M. Liu, and H. R. Brand, Rheol. Acta 41, 375 (2002).

[7] L. Noirez, Phys. Rev. E 72, 051701 (2005); H. Mendil, P. Baroni, and L. Noirez, Europhys. Lett. 72, 983 (2005); Eur. Phys. J. E 19, 77 (2006).

[8] W. Renz and M. Warner, Phys. Rev. Lett. 56, 1268 (1986); X. J. Wang and M. Warner, J. Phys. A 20, 713 (1987).

[9] L. Noirez, P. Keller, and J. P. Cotton, Liq. Cryst. 18, 129 (1995).

[10] S. F. Rubin et al., Macromolecules 28, 3521 (1995); R. M. Kannan et al., Macromolecules 26, 2050 (1993); R. H. Colby et al., Liq. Cryst. 13, 233 (1993).

[11] J. L. Gallani, L. Hilliou, P. Martinoty, and P. Keller, Phys. Rev. Lett. 72, 2109 (1994); P. Martinoty et al., Macromolecules 32, 1746 (1999); D. Collin and P. Martinoty, Physica (Amsterdam) 320A, 235 (2003).

[12] V. Castelletto, L. Noirez, and P. Vigoureux, Europhys. Lett. 52, 392 (2000).

[13] J. Higgins and H. Benoît, Polymers and Neutron Scattering (Clarendon Press, Oxford, England, 1994).

[14] PXY, available at the LLB by G. Pépy.

[15] L. Noirez, Europhys. Lett. 46, 728 (1999).

[16] J.D. Ferry, Viscoelastic Properties of Polymers (Wiley, New York, 1980), 3rd ed.

[17] N. Grassie et al., J. Polym. Sci., Polym. Chem. Ed. 9, 931 (1971).

[18] A. Vewerka, K. Viertler, D. Vlassopoulos, and F. Stelzer, Rheol. Acta 40, 416 (2001).

[19] U. Tracht et al., Phys. Rev. Lett. 81, 2727 (1998).

[20] A. S. Bakai, J. Non-Cryst. Solids 307, 623 (2002).

[21] L. Noirez, Phys. Rev. Lett. 84, 2164 (2000).

[22] S. I. Ben Abraham and P. Ostwald, Mol. Cryst. Liq. Cryst. 93, 383 (1983); P. Ostwald and M. Kléman, J. Phys. (Paris), Lett. 43, 411 (1982); R. Ribotta and G. Durand, J. Phys. (Paris) 38, 179 (1977). 